AN OPTIMIZATION MODEL FOR SELECTING TRAINING COURSE LOCATIONS, U.S. GEOLOGICAL SURVEY

By Timothy A. Cohn and William G. Baier

U.S. GEOLOGICAL SURVEY

Open-File Report 93-123

Reston, Virginia

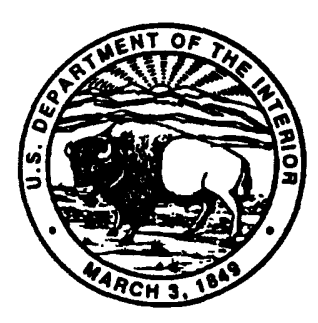




\section{U. S. DEPARTMENT OF THE INTERIOR \\ BRUCE BABBITT, Secretary \\ U.S. GEOLOGICAL SURVEY}

DALLAS L. PECK, Director

For additional information write to:

Chief, Branch of Systems Analysis

U.S. Geological Survey

410 National Center

Reston, Virginia 22092
Copies of this report can be purchased from:

U.S. Geological Survey Books and Open-File Reports Box 25425, Federal Center Denver, Colorado 80225 


\section{CONTENTS}

\section{Page}

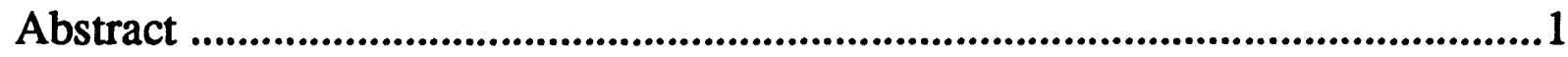

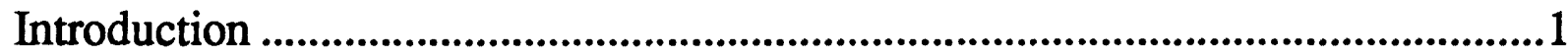

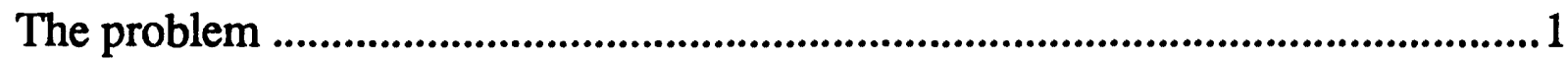

Assumptions and limitations of the model ...............................................3

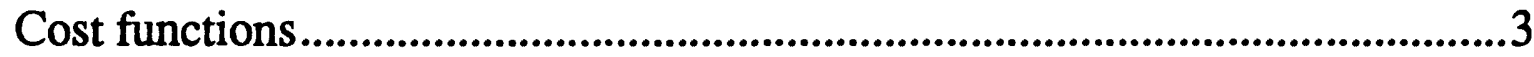

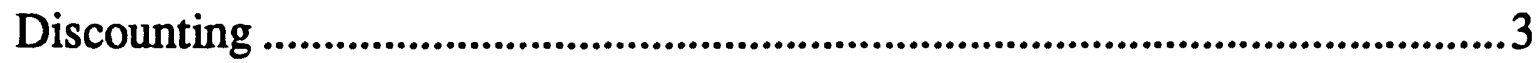

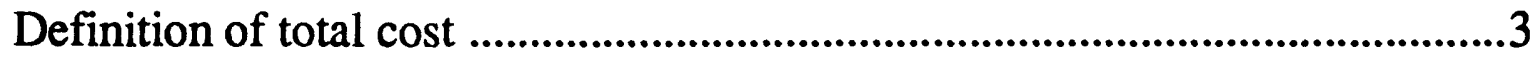

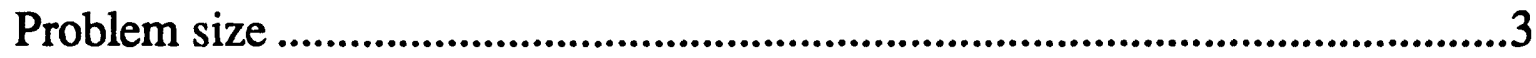

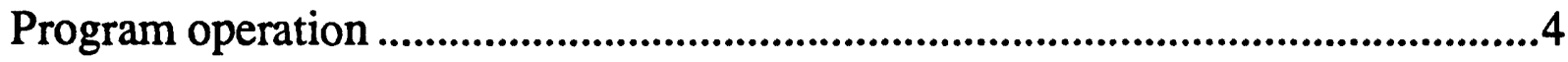

Program input data file ................................................................................4

Running the program ...............................................................................6

Interpretation of program output files ...........................................................6

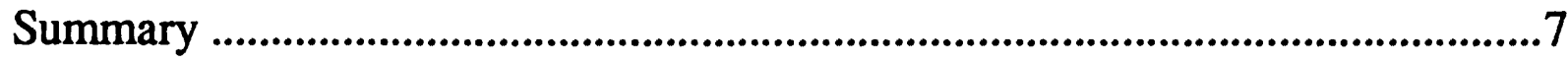

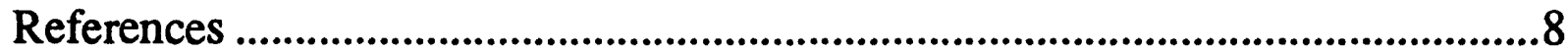

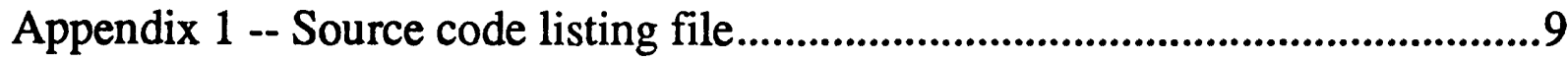

Appendix 2 -- Sample output file ........................................................................14

\section{TABLES}

Table

1. Input data format for the program..................................................5

2. Sample input data table for the program .........................................5 


\title{
AN OPTIMIZATION MODEL FOR SELECTING TRAINING COURSE LOCATIONS, U. S. GEOLOGICAL SURVEY
}

\author{
By Timothy A. Cohn and William G. Baier
}

\begin{abstract}
This report describes a computer program, named KATALASE, originally designed to assist the Northeastern Region, Water Resources Division, U. S. Geological Survey, in minimizing training course costs. The program can be used by planners, at any level, to determine the least expensive location for meetings and training events. KATALASE employs a combination of enumeration and linear programming to minimize a cost function subject to constraints on number of attendees and site capacities. Up to twenty-five potential sites can be analyzed to determine which location, or set of locations, will support the desired training at the lowest cost. The program output provides total cost, site(s), and attendee routing schemes.
\end{abstract}

\section{INTRODUCTION}

In the Northeastern Region, Water Resources Division, U. S. Geological Survey, all new employees are required to take a series of training classes. Each of these classes can be taught at a number of different sites throughout the region. The desire to minimize overall costs raises two questions:

1) At which site(s) should the training classes be offered?

2) To which training site should each new employee be sent? A computer program called KATALASE answers these questions. KATALASE can also be used to solve other similar linear network problems [Bradley and others, 1977].

This report provides instructions for using KATALASE, and describes its limitations and underlying assumptions. Discussions of input data file format, program execution, and interpretation of output files are provided. For those interested, a brief discussion of the program design is included.

\section{THE PROBLEM}

Assume we have a number of offices wishing to send new employees to training, and a number of sites that can provide training. The source sites are denoted $\left\{S_{1}, \ldots, S_{N S N T}\right\}$, where NSNT is the total number of offices wishing to 
send new employees to training. The sites that can provide training are denoted $\left\{\mathrm{TS}_{1}, \ldots, \mathrm{TS}_{\mathrm{NPTS}}\right\}$, where NPTS is the total number of sites that can provide training.

KATALASE finds optimal solutions for one training course at a time. The optimal solution may suggest teaching the course at multiple training sites. If several courses need to be scheduled, KATALASE must be run separately for each one.

The constraints and costs can be formulated as an optimization problem with linear constraints and linear objective function [see Bradley and others, 1977, p. 310]:

$$
\begin{aligned}
& \text { minimize } \mathrm{C}=\Sigma_{\mathrm{j}} \mathrm{N}_{\mathrm{j}} \mathrm{f}_{\mathrm{j}}+\Sigma_{\mathrm{i}} \Sigma_{\mathrm{j}} \mathrm{T}_{\mathrm{ij}} \mathrm{c}_{\mathrm{ij}} \\
& \text { subject to: } \quad \Sigma_{j} T_{i j}=d_{i} \quad i=1, \text { NSNT } \\
& \Sigma_{i} T_{i j} \leq N_{j} q_{j} \quad j=1, \text { NPTS } \\
& \Sigma_{\mathrm{i}} \mathrm{T}_{\mathrm{ij}} \geq \mathrm{N}_{\mathrm{j}} \mathrm{l}_{\mathrm{j}} \quad \mathrm{j}=1 \text {, NPTS } \\
& \Sigma_{\mathrm{j}} \mathrm{N}_{\mathrm{j}} \leq \mathrm{NT} \quad \mathrm{i}=1, \mathrm{NSNT}
\end{aligned}
$$

where:

$$
\begin{aligned}
& C=\text { total cost, } \\
& \Sigma_{j}=\text { summation over }\left\{T_{j}\right\}, \\
& \Sigma_{i}=\text { summation over }\left\{S S_{i}\right\} \\
& N_{j}=\text { number of times training is held at } T S_{j}, \\
& N T=\text { maximum number of training classes permitted in the solution } \\
& f_{j}=\text { fixed costs of training at } T S_{j}, \\
& T_{i j}=\text { number of attendees from } S S_{i} \text { attending training at } T S_{j}, \\
& c_{i j}=\text { travel cost for one attendee from } S S_{i} \text { to attend training at } T S_{j}, \\
& d_{i}=\text { number of employees needing training from } S S_{i}, \\
& q_{j}=\text { maximum number of attendees at } T S_{j}, \text { and } \\
& q_{j}=\text { minimum number of attendees at } T S_{j} .
\end{aligned}
$$

KATALASE ensures that demand and capacity constraints are fulfilled while cost is minimized. In short, KATALASE finds each attendee space in the class and ensures each class is not overbooked, while at the same time, minimizing total costs.

\section{The KATALASE Algorithm}

The KATALASE algorithm consists of four stages:

1) All possible combinations of training sites are listed;

2) For each combination of training sites, a linear program is used to find the least cost allocation of attendees to the training sites;

3) The least cost solution for each combination is recorded;

4) The combinations are ranked by cost. 
This four-stage method guarantees integer solutions while avoiding more complicated integer techniques like "branch and bound" [Bradley and others, 1977, p. 387].

\section{ASSUMPTIONS AND LIMITATIONS OF MODEL}

\section{Cost Functions}

The first assumption is that fixed costs and travel costs are constant. This program does not recognize seasonal changes in airline, hotel, or other rates. If users wish to take seasonal rate changes into account, they may consider multiple entries for each site. For example, if fixed costs for Reston (U. S. Geological Survey National Center) are different in the summer than in the winter, then the user may wish to enter Reston(summer) and Reston(winter) as separate sites.

\section{Discounting}

The program does not permit non-linear cost functions. For example, a convention center might offer a discount if an organization commits to having more than one meeting per year at that facility. The program does not make use of this information.

\section{Definition of Total Cost}

The program is designed to minimize total costs for an event. For an event involving multiple organizations, the optimal solution may not minimize costs for each organization. If an organization hosting a multiple-organization event wishes to minimize its own cost, disregarding others' costs, only that organization's costs should appear in the input data. The fixed costs and travel costs for the other organizations should be set to zero.

Fixed costs and travel costs are, respectively, the sum of all costs associated with an event and the costs associated with transporting an attendee to the event. Determining the actual values for fixed cost and travel cost is the responsibility of the user. The optimal solution will be a function of these costs.

\section{Problem Size}

The number of combinations listed in stage 1 of the KATALASE algorithm ( $\mathrm{N}_{\text {com }}$ ) depends on NPTS and NT according to the following formula:

$$
\mathrm{N}_{\text {com }}=\left(\begin{array}{c}
\mathrm{NPTS}+\mathrm{NT} \\
\mathrm{NT}
\end{array}\right)=\frac{(\mathrm{NPTS}+\mathrm{NT}) !}{\mathrm{NPTS} ! \mathrm{NT} !}
$$

Because $\mathrm{N}_{\text {com }}$ can become very large for large values of NPTS and NT, KATALASE requires that:

1) NPTS not exceed 25; and

2) NT not exceed 10. 
The user should choose a value for NT no larger than the maximum number of times that the course will be taught. Larger values of NT will substantially increase the amount of computer time required to solve the problem, and may result in solutions which suggest teaching the course more than the desired number of times. However, selecting values of NT which are too small may prevent KATALASE from considering lower-cost solutions which involve more classes. If NT is too small a feasible solution will not exist and the program output will include a string of asterisks for the minimum-cost solution.

KATALASE automatically adds one potential site with capacity, fixed cost, and travel costs equal to zero. This site allows the optimal solution to include fewer than NT sites. Combinations can contain the same training site multiple times. Permutations containing the same sites in different orders are considered only once.

\section{PROGRAM OPERATION}

\section{Program Input Data File}

The required input data format for the KATALASE program is shown in Table 1. Table columns are tab-separated and table rows are separated by a carrage return. Input files can be created using any spreadsheet or editor software which allows tab separation output (e.g. Excel1, Lotus-123, Tactician). The input file must include the bold italicized words (For example, Minimum and $q l$ ). These maintain data alignment, insuring correct interpretation of input data.

Blank spaces are not permitted between characters occupying the same cell. This is important when entering multiple word names. (New York entered as two words is not permitted. However, alternatives such as NewYork, New_York, and New.York are permitted).

An example of an input file containing information for 5 potential training sites (NPTS $=5$ ) and 7 sites needing training (NSNT $=7$ ) is shown in Table 2. Note that the potential training sites and the sites needing training can be different. 5 employees from Baltimore need training (dBaltimore $=5)$. For a course held in Boston, the minimum capacity is 15 (qlBoston $=15$ ) and the maximum capacity is 32 (qBoston=32). The fixed cost for an event held in Boston is $\$ 2487.00$ $\left(f_{\text {Boston }}=\$ 2487.00\right)$. The travel cost for one employee traveling from Baltimore to train at Boston is $\$ 895.00$ (cBaltimore,Boston $=\$ 895.00$ ).

1 The use of brand names in this report is for identification purposes only, and does not constitute endorsement by the U. S. Geological Survey. 
Table 1.--Input data format for the KATALASE program

[NPTS, number of potential training sites; NSTN, number of sites needing training; TS, potential training site; q, maximum capacity; ql, minimum capacity; f, fixed cost; SS, source site; c, travel cost]

\begin{tabular}{|c|c|c|c|c|c|c|}
\hline NPTS & NPTS & & & & & \\
\hline NSNT & NSNT & $\overline{T S 1}$ & $\overline{T_{2}}$ & TS3 & $\ldots$ & TS $_{\text {NPTS }}$ \\
\hline Capacity & $q$ & $\mathrm{q}_{1}$ & $\overline{q_{2}}$ & $\overline{q_{3}}$ & $\ldots$ & $q_{\text {NPTS }}$ \\
\hline Minimum & $q l$ & $\mathrm{ql}_{1}$ & $\mathrm{ql}_{2}$ & $\mathrm{ql}_{3}$ & $\ldots$ & $\mathrm{ql}_{\mathrm{NPTS}}$ \\
\hline Fixed_cost & $\bar{f}$ & $\mathrm{f} 1$ & $f_{2}$ & f3 & $\ldots$ & $f_{\text {NPTS }}$ \\
\hline & Demand & & & & & \\
\hline $\mathrm{SS}_{1}$ & d1 & $\mathrm{c} 11$ & $\mathrm{c12}$ & $\mathrm{c} 13$ & $\ldots$ & c1NPTS \\
\hline $\mathrm{SS}_{2}$ & $\mathrm{~d}_{2}$ & $\mathrm{c} 21$ & c12 & c13 & $\ldots$ & c2NPTS \\
\hline SS3 & d3 & c31 & $\mathrm{c12}$ & $\mathrm{c} 13$ & $\ldots$ & C3NPTS \\
\hline$\ldots$ & $\ldots$ & $\ldots$ & $\ldots$ & $\ldots$ & $\ldots$ & $\ldots$ \\
\hline SS $_{\text {NSNT }}$ & $\mathrm{d}_{\mathrm{NSNT}}$ & $c_{\text {NSNT } 1}$ & $\mathrm{c}_{\mathrm{NSNT}} 2$ & $\mathrm{c}_{\mathrm{NSNT}} 3$ & $\ldots .$. & $\mathrm{c}_{\text {NSNT,NPTS }}$ \\
\hline
\end{tabular}

Table 2.--Sample input data table for the KATALASE program

\begin{tabular}{|l|l|l|l|l|l|l|}
\hline NPTS & 5 & & & & & \\
\hline NSNT & 7 & Hartford & Champaign & Louisville & Reston & Boston \\
\hline Capacity & $q$ & 30 & 24 & 24 & 35 & 32 \\
\hline Minimum & $q l$ & 15 & 15 & 15 & 15 & 15 \\
\hline Fixed_cost & $f$ & 2499.00 & 3465.00 & 2727.00 & 0.00 & 2487.00 \\
\hline & Demand & & & & & \\
\hline Hartford & 1 & 0.00 & 937.00 & 987.00 & 833.00 & 499.00 \\
\hline Champaign & 4 & 1041.00 & 0.00 & 779.00 & 1155.00 & 1025.00 \\
\hline Inianapolis & 5 & 1037.00 & 685.00 & 775.00 & 863.00 & 779.00 \\
\hline Louisville & 4 & 1067.00 & 755.00 & 0.00 & 909.00 & 841.00 \\
\hline Baltimore & 5 & 853.00 & 897.00 & 741.00 & 514.10 & 895.00 \\
\hline Boston & 30 & 447.10 & 869.00 & 709.00 & 829.00 & 0.00 \\
\hline Lansing & 5 & 1109.00 & 757.00 & 845.00 & 1223.00 & 1093.00 \\
\hline
\end{tabular}




\section{Running the Program}

To start KATALASE the user should:

1) Copy the program's executable file, katalase.out, into his/her directory;

2) Create the input data file, which should be checked for tab separation between entries, carrage returns between rows, and properly-placed Dummy variables;

3) Type katalase.out

The program will prompt the user for the input data file name. Entering an improper file name or problems with the input data file format may cause the program to stop execution. If this happens, a FORTRAN error statement will appear.

Next, the user will be asked to enter the maximum number of sites to be used in the solution (NT). If the planned event is a single occurrence event, the user's response should be 1 . If the planned event is a series of meetings or multiple offerrings of the same training course, then the number enterred should reflect the maximum number of meetings or training course offerings.

The final prompt asks for the number of solutions to show in full $\left(\mathrm{N}_{\text {show }}\right)$. The complete optimal solution will be saved for the $\mathrm{N}_{\text {show }}$ least-cost solutions. Regardless of the response, the optimal cost and training sites for all combinations will be saved to the output file. No results are printed to the screen.

\section{Interpretation of Program Output Files}

The KATALASE program output is automatically saved in the file results_input filename. A sample output file is presented in Appendix 2. Output files contain; input file name, output file name, NPTS, NT, NSNT, an echo of the input data file, and a listing of optimal solutions. Examining the echoed data is recommended.

The sample output file in Appendix 2 gives complete results for the first three optimal solutions. The remaining results only contain cost and sites. (The sample output was edited to show only the first and last ten solutions.) In this sample the maximum number of sites was set at three (NT=3).

The results for the optimal solution suggest that:

1. The minimum total cost for this training is $\$ 20634.00$.

2. The training sites will be Louisville and Boston (only two sites are needed).

3. 31 employees will attend training in Boston. 30 will come from Boston, 1 will come from Hartford. 


\section{SUMMARY}

In summary, KATALASE is an optimization program designed to assist planners in scheduling training courses. The user specifies costs associated with each potential training site. KATALASE then identifies the minimum-cost solution, costs of alternate solutions, and provides attendee routing schemes. 


\section{REFERENCES}

Bradley, S. P., A. C. Hax and T. L. Magnanti, (1977), Applied Mathematical Programming, Addison-Wesley, Reading, Massachusetts, 716 pp. 


\section{APPENDIX 1, SOURCE CODE LISTING FILE}

program katalase

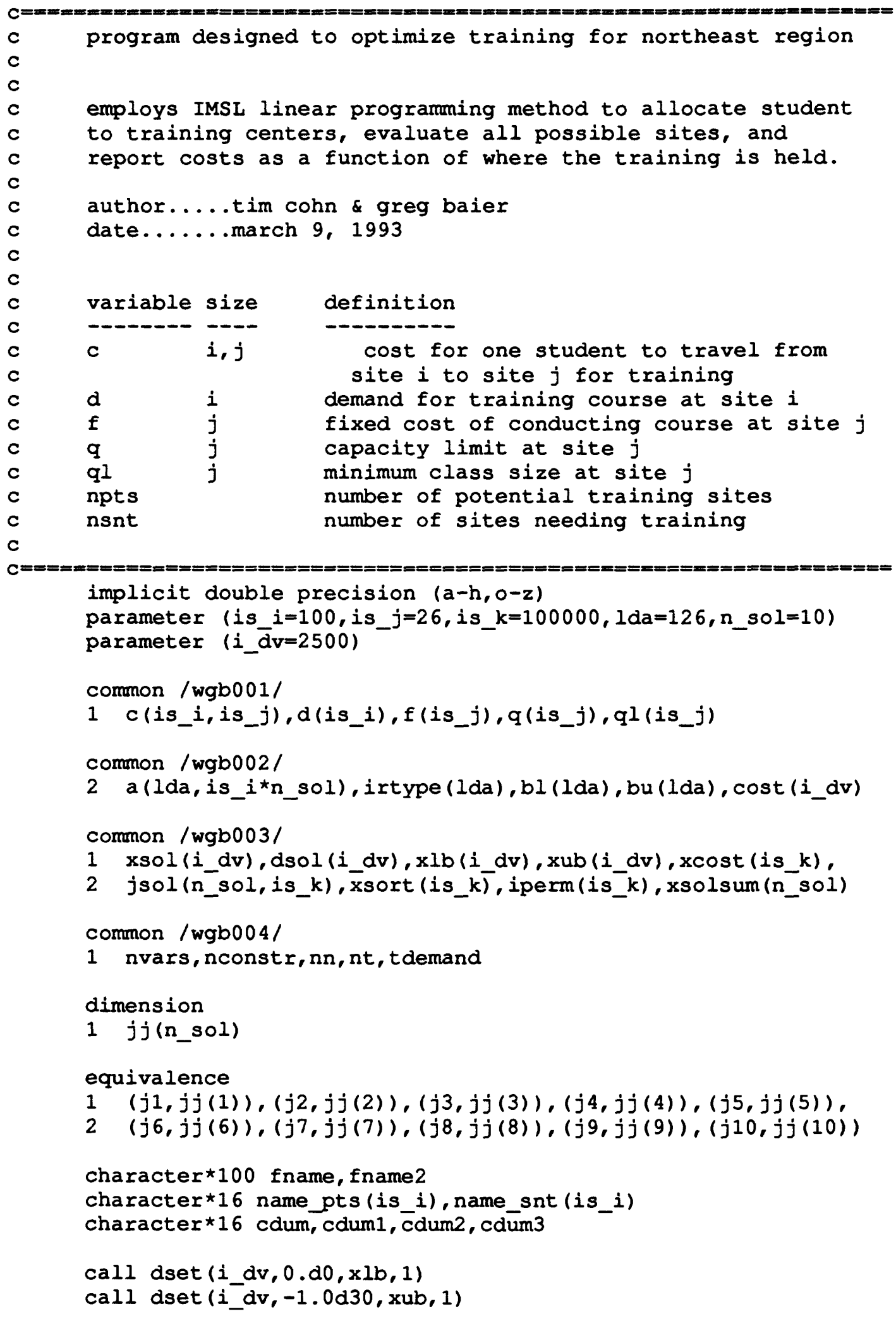


call dset (1da, 9999.d0,bu, 1)

call dset (lda, $-9999 . d 0, b l, 1$ )

C

C

C

C

C

C

c

C

C

c

C

C

c

C

C

c

c

C

read in the input data for travel costs, fixed costs, demand, capacity

write $(*, *)$ ' enter the data file name'

read $(*, '(a 100) ')$ fname

open (unit $=11, f i l e=f n a m e, ~ s t a t u s=' o l d '$ )

write (fname2,' (a8, a92)') 'results ', fname (1:92)

open (unit $=12, f i l e=f$ name 2 , status=' 'new')

Get maximum number of training sites to consider simultaneously

write $(*, *)$ ' enter max. no. training locations allowed in solution' $\operatorname{read}(*, *) \mathrm{NT}$

if (NT .gt. n sol) then

write $(*, * \overline{)}$ ' maximum number of training locations is:', n_sol stop

endif

write $(*, *)$ ' enter the number of solutions to present in full'

$\operatorname{read}(*, *)$ ncheck

write out banner for page 1

write $(12,110)$

format $(/, t 20$, 'LP OPTIMIZATION FOR TRAINING LOCATIONS')

write $(12,111)$

format (t20,' Timothy A. Cohn and William G. Baier')

write $(12,112)$

format (t20,' version 1.0, March 9, 1993',//)

write $(12,114)$ fname

format(' Input File Name: ',a100)

write $(12,115)$ fname2

format(" Output File Name: ', a100)

Read number of training sites; add dummy null site at end

$\operatorname{read}(11, *)$ cdum 1, npts

npts $=$ npts+1

if (npts.gt. is i) then

write $(*, *)$ ' number of sites exceeds program capability' stop

endif

write $(12, '(a 36, i 6)$ ') ' No. Potential Training Sites (NPTS) ', npts-1

write $\left(12, '(a 36, i 6)^{\prime}\right)$ ' No. Sites in solution (NT) ,NT

Read training site names, nsnt

$\operatorname{read}(11, *) \quad c d u m 1, n s n t,($ name_pts $(k), k=1$, npts-1)

name pts (npts) = '----'

write $(12, '(a 36, i 6)$ ') ' No. Sites Needing Training (NSNT) ', nsnt

read in capacity at each training site, fixed costs

write (12,'(//,t33, 'Possible Training Sites'')')

write $(12,122)$ (name_pts $(k)(1: 7), k=1$, npt s-1)

format $(/, 9(t 24,7(1 x, a 7) /))$

$\operatorname{read}(11, \star) \operatorname{cdum}, \operatorname{cdum} 2,(q(j), j=1, \operatorname{npts}-1)$ 
C

C

Read in cost data

C

c continue

c

c

C

c

c

40 continue

$\mathrm{NN}=\mathrm{nsnt}$

set up constraint matrices

nconstr $=\mathrm{NT}+\mathrm{NN}$

nvars $=\mathrm{NT} \star \mathrm{NN}$

call dset $\left(l d a \star_{n}\right.$ sol $\left.{ } i s \_i, 0 . d 0, a, 1\right)$

do $40 \quad i=1$, NN

$b l(i)=d(i)$

$b u(i)=d(i)$

irtype $(i)=0$

do $40 j=1, N T$

continue

$a\left(i, i+N^{\star}(j-1)\right)=1 . d 0$

c

$$
\text { do } \begin{aligned}
50 \quad & j=1, N T \\
& i r t y p e(N N+j)=3 \\
\text { do } & 50 \quad i=1, N N \\
& a(j+N N, i+N N *(j-1))=1 . d 0
\end{aligned}
$$

continue

c

$$
\text { ict }=0
$$

c

C

c

Begin major do-loops

do $30 j 1=1$, npts

do $30 j 2=\max (j 1$, sign (npts, 1-nt)), npts

do $30 j 3=\max (j 2, \operatorname{sign}(n p t s, 2-n t))$, npts 
do $30 j 4=\max (j 3, \operatorname{sign}$ (npts, 3-nt)), npts

do $30 j 5=\max (j 4$, sign (npts, 4-nt)), npts

do $30 j 6=\max (j 5$, sign (npts, 5-nt)), npts

do $30 j 7=\max (j 6$, sign (npts, $6-n t))$, npts

do $30 j 8=\max (j 7$, sign (npts, $7-n t)$ ), npts

do $30 j 9=\max (j 8$, sign (npts, $8-n t))$, npts

C

do $30 j 10=\max (j 9, \operatorname{sign}(n p t s, 9-n t))$, npts

C

ict $=i c t+1$

if (ict.gt. is_k) then

write $(*, *) T$ number of cases exceeds program capability' stop

endif

call runit (jj, $x \operatorname{cost}(i c t))$

do $30 k=1$, nt

$j \operatorname{sol}(k, i c t)=j j(k)$

30

continue

C

Sort results with IMSL dsvrgp; prepare output

c

do $65 \mathrm{k}=1$, ict

$\operatorname{iperm}(k)=k$

continue

call dsvrgp (ict, $x$ cost, xsort, iperm)

C

C

Output Results

c

113

write $(12,113)$

format $(/ / / / / /, t 31$, OPTIMAL SOLUTIONS,$/ / /)$

write $(12,110)$

write $(12,111)$

write $(12,112)$

write $(12,116)$

116 format (' Solutions listed in order of increasing cost')

write $(12,117)$

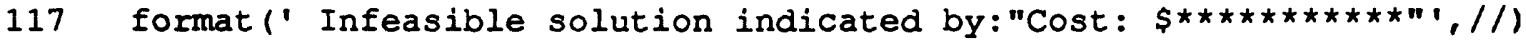

do $70 i=1$, ict

index $=i \operatorname{perm}(i)$

write $(12,100) i, x \operatorname{cost}$ (index), (name pts (jsol ( $k, i n d e x)), k=1, N T$ )

100

format (i4,' Cost: $\$$ ',f11.2,6x,10(a16))

if ( $i$.le. ncheck) then

call dset (NT, 0. d0, xsolsum, 1 )

call runit (jsol (1, index), tcost)

do $60 j t=1$, nsnt

write $(12,101)$ name snt $(j t),($ int $(x \operatorname{sol}(j t+(k-1) \star N N)), k=1, N T)$

101

60

format $(6 x, a 16,3 x, 1 \overline{0}(i 9,7 x))$

do $60 \mathrm{k}=1$, NT

ind $\quad=j t+(k-1) \star N N$

xsolsum $(k)=$ xsolsum $(k)+x s o l(i n d)$

continue

write $(12,101)$ 'Total Attendees', (int (xsolsum (k)), k=1,NT)

endif write $(12, '(/ /)$ ')

70 continue

stop

end 
C

subroutine runit $(j j, t$ cost $)$

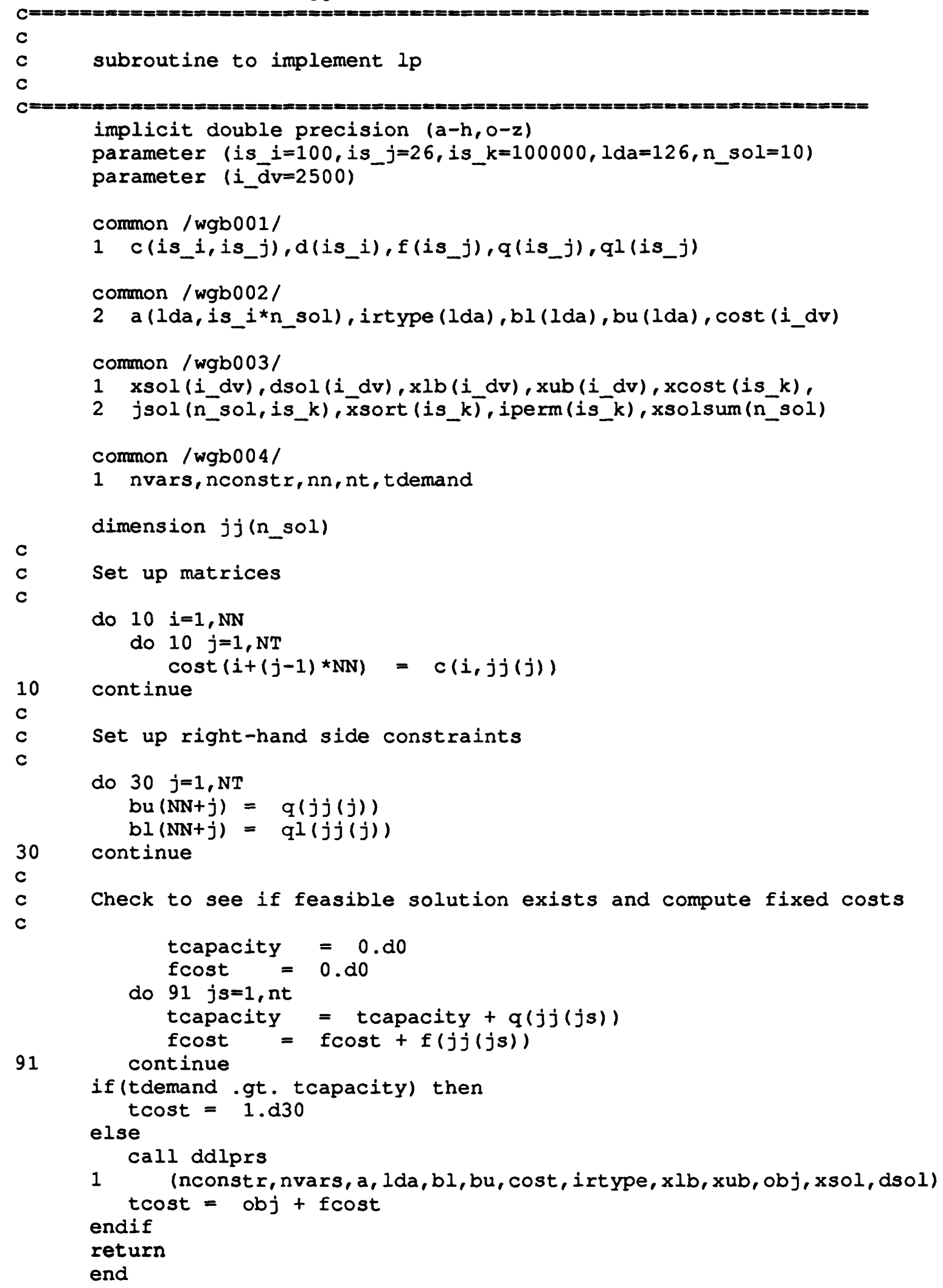




\title{
APPENDIX 2, SAMPLE OUPUT FILE
}

\author{
LP OPTIMIZATION FOR TRAINING LOCATIONS \\ Timothy A. Cohn and William G. Baier \\ version 1.0, March 9, 1993
}

\begin{abstract}
Input File Name: sample.input
Output File Name: results_sample.input

No. Potential Training Sites (NPTS) 5

No. Sites in Solution (NT)

No. Sites Needing Training (NSNT) 7
\end{abstract}

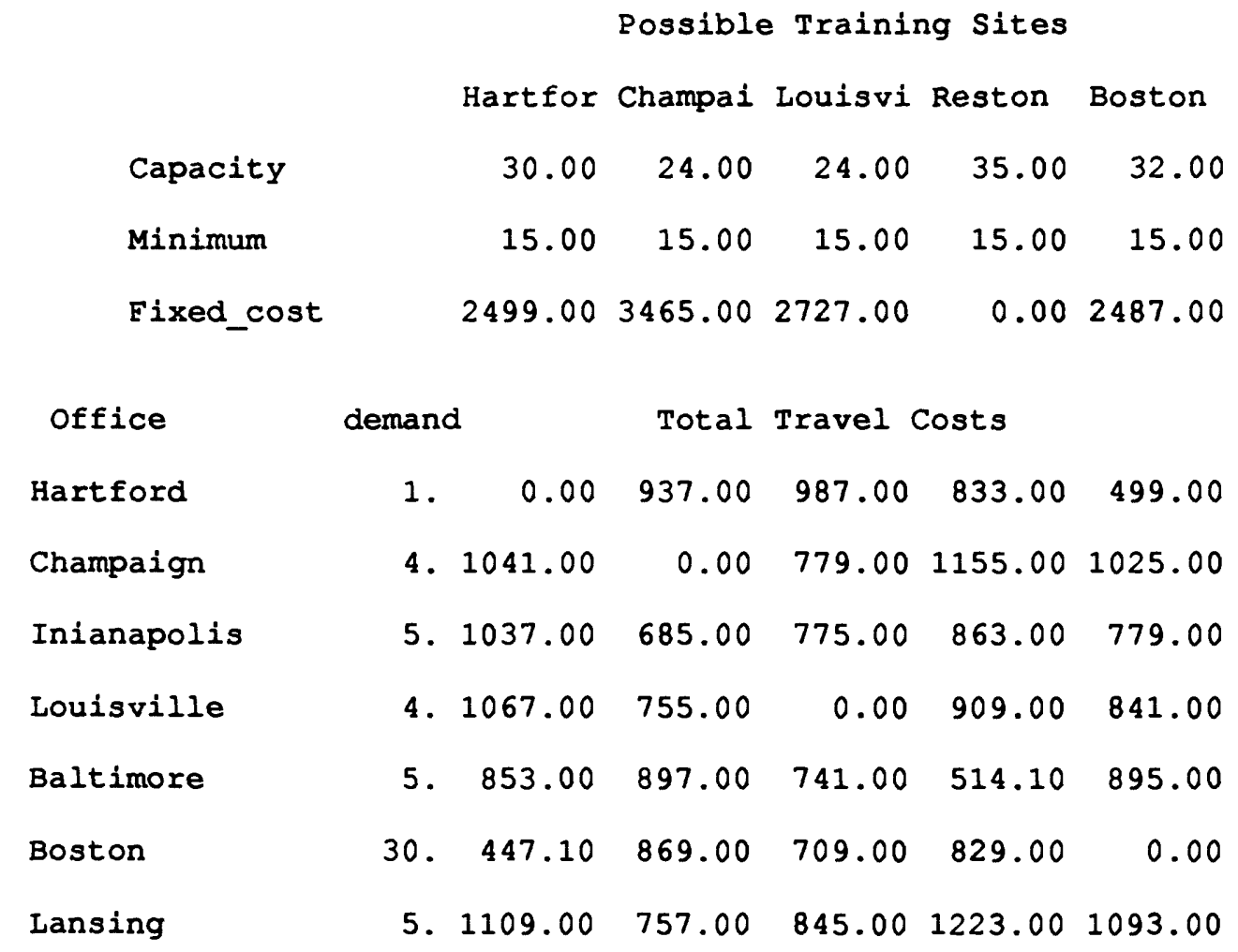

OPTIMAL SOLUTIONS

LP OPTIMIZATION FOR TRAINING LOCATIONS

Timothy A. Cohn and William G. Baier version 1.0, March 9, 1993 
Solutions listed in order of increasing cost

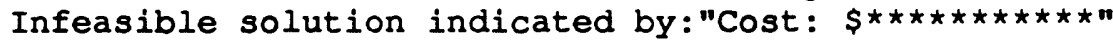

1 Cost: \$ 20634.00

Hartford

Champaign

Inianapolis

Louisville

Baltimore

Boston

Lansing

Total Attendees

Louisville
0
4
5
4
5
0
5
23

Boston

$\begin{array}{rr}1 & 0 \\ 0 & 0 \\ 0 & 0 \\ 0 & 0 \\ 0 & 0 \\ 30 & 0 \\ 0 & 0 \\ 31 & 0\end{array}$

2 Cost: \$ 21164.00

Hartford

Champaign

Inianapolis

Louisville

Baltimore

Boston

Lansing

Total Attendees

Champaign
0
4
5
4
4
0
5
22

Boston

1

0

0

0

1

30

0

32

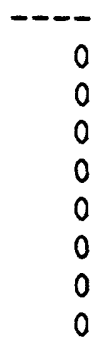

3 Cost: \$ 23121.00

Hartford

Champaign

Inianapolis

Louisville

Baltimore

Boston

Lansing

Total Attendees

Louisville
0
4
5
4
5
0
5
23

Boston

Boston

0
0
0
0
0
16
0
16

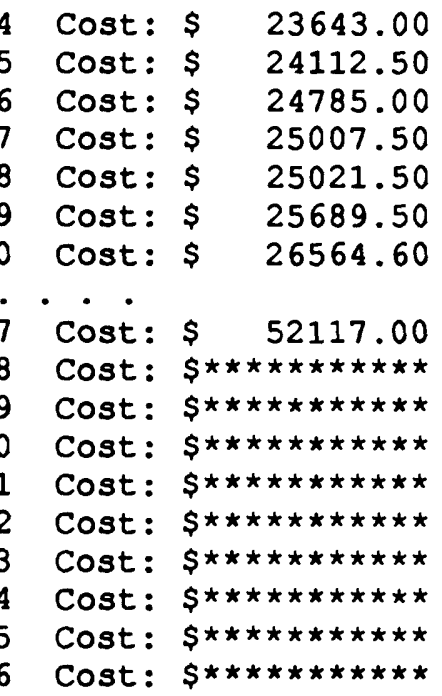

Champaign

Reston

Champaign

Louisville

Champaign

Reston

Hartford

Champaign

Champaign

Champaign

Louisville

Champaign

Reston

Louisville

Hartford

Boston
Boston

Boston

Louisville

Reston

Reston

Boston

Champaign

Champaign

Louisville

Louisville

Champaign

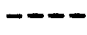

---

$-\cdots$

$--$
Boston

----

Boston

Boston

Boston

Boston

Boston

Champaign

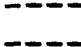

$---$

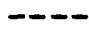

----

---

- - 